\title{
Competitive inhibition of three novel bacteria isolated from faeces of breast milk-fed infants against selected enteropathogens
}

\author{
Sergio Muñoz-Quezada ${ }^{1}$, Miriam Bermudez-Brito ${ }^{1}$, Empar Chenoll $^{2}$, Salvador Genovés ${ }^{2}$, \\ Carolina Gomez-Llorente ${ }^{1}$, Julio Plaza-Diaz ${ }^{1}$, Esther Matencio ${ }^{3}$, María José Bernal ${ }^{3}$, \\ Fernando Romero ${ }^{3}$, Daniel Ramón ${ }^{2}$ and Angel Gil ${ }^{1 *}$ \\ ${ }^{1}$ Department of Biochemistry and Molecular Biology II, Institute of Nutrition and Food Technology "José Mataix", Biomedical \\ Research Centre, University of Granada, Avenida del Conocimiento s/n, 18100 Armilla, Granada, Spain \\ ${ }^{2}$ Department of Food Biotechnology, Biópolis S.L., Parc Científic Universitat de València, C/Catedrático Agustín Escardino 9, \\ Edificio 2, 46980 Paterna, Valencia, Spain \\ ${ }^{3}$ Hero Global Technology Centre For Infant Nutrition, Hero Group, Avenida Murcia 1, 30820-Alcantarilla, Murcia, Spain
}

\section{Abstract}

Numerous in vitro and in vivo studies conducted using different probiotic micro-organisms have demonstrated their ability to interfere with the growth and virulence of a variety of enteropathogens. The reported beneficial effects of the use of probiotics to complement antibiotic therapy or prevent diarrhoea or gastrointestinal infection in infants have increased in recent years. In the present study, we demonstrated the capacity of supernatants obtained from three novel probiotics (Lactobacillus paracasei CNCM I-4034, Bifidobacterium breve CNCM I- 4035 and Lactobacillus rhamnosus CNCM I-4036) isolated from the faeces of breastfed infants to inhibit the growth of enterotoxigenic and enteropathogenic (EPEC) bacteria, such as Escherichia coli, Salmonella and Shigella. To assess their potential antimicrobial activity, the 17 and $24 \mathrm{~h}$ cell-free supernatants broth concentrates (10X) having 1, 2 or $4 \%$ of the three probiotics were incubated with EPEC bacteria strains. After $17 \mathrm{~h}$ of co-culture, the supernatants were able to inhibit the growth of E. coli, Salmonella and Shigella up to 40, 55 and $81 \%$, respectively. However, the inhibitory capacity of some supernatants was maintained or completely lost when the supernatants ( $\mathrm{pH}$ 3.0) were neutralised ( $\mathrm{pH}$ 6.5). Overall, these results demonstrated that L. paracasei CNCM I-4034, B. breve CNCM I-4035 and L. rhamnosus CNCM I-4036 produce compounds that exhibited strain-specific inhibition of enterobacteria and have the potential to be used as probiotics in functional foods.

Key words: Bacterial competition; Bifidobacterium breve; enteropathogens; Lactobacillus paracasei; Lactobacillus rhamnosus; probiotics.

Probiotics are defined as living micro-organisms that confer a health benefit to the host when administered in adequate amounts $^{(1)}$. One of the most frequent health claims for probiotics concerns the putative reduction and prevention of infectious disease in the gastrointestinal tract. The regular intake of probiotic micro-organisms has been demonstrated to prevent several infectious diseases, allergic disorders, diarrhoea and inflammatory diseases, such as inflammatory bowel disease ${ }^{(2)}$.

Bacteria are present in the food, water and environment, promoting gastrointestinal tract susceptibility to different types of infection, which produce a variety of illnesses in human subjects worldwide. Escherichia coli strains possess variable mechanisms of pathogenesis, while enterotoxigenic (ETEC) strains produce enterotoxin and enteropathogenic (EPEC) strains that adhere to epithelial cells; the latter type of infection remains a formidable cause of diarrhoeal illnesses. Zoonotic pathogens and those transmitted through faecal-oral contact, such as Salmonella and Shigella, respectively, constitute an important public health problem, especially in developing countries with substandard hygiene and unsafe water supplies. Antibiotics represent the first line of treatment, but the use of antibiotics causes an imbalance in the complex ecosystem of the human gastrointestinal tract. Thus, therapeutic alternatives to prevent or complement antibiotic therapy are currently being assessed. In this respect, the use of probiotics is a promising tool to prevent EPEC infections.

Several studies have demonstrated antimicrobial activity by lactobacilli and bifidobacteria against gastrointestinal microbial pathogens ${ }^{(3)}$. The mechanism underlying these effects of probiotics are largely unknown but are likely to be multifactorial, including the reduction of luminal $\mathrm{pH}$, competition for nutritional sources, inhibition of adhesion to

Abbreviations: EPEC, enteropathogenic; ETEC, enterotoxigenic.

*Corresponding author: A. Gil, fax +34958 819132, email agil@ugr.es 
epithelial cells, stimulation of the host immune system and the production of organic acids, bacteriocin or bacteriocin-like substances $^{(4)}$.

Some probiotics produce metabolites that inhibit the growth of bacteria and fungi ${ }^{(5,6)}$ and have been used to prevent intestinal pathogenic infections, such as those caused by Salmonella, Shigella, E. coli, Listeria and Helicobacter pylori ${ }^{(7-11)}$. Due to their reported health benefits and the large number of novel probiotic strains, they have become attractive candidates for incorporation into functional foods and food products.

Among all probiotic bacteria, those isolated from the faeces of exclusively breastfed infants are of special interest due to lactic acid bacteria predominance, and these strains also seem to provide protection against enteric and systemic disorders caused by bacterial pathogens ${ }^{(12,13)}$.

Recently, we reported the selection and characterisation of three probiotic strains (Lactobacillus paracasei CNCM I-4034, Bifidobacterium breve CNCM I-4035 and Lactobacillus rhamnosus CNCM I-4036) isolated from the faeces of breastfed infants $^{(11)}$. We demonstrated that these strains exhibited probiotic potential, survival in gastrointestinal conditions and adhesion to intestinal cells, and they were also innocuous to human health. Their safety status has been confirmed by sensitivity to antibiotics, toxicology, the absence of undesirable metabolites and acute ingestion studies in immunocompetent and immunosuppressed mice. Furthermore, antimicrobial activity against human rotavirus and Listeria monocytogenes has also been described for these probiotics.

The aim of the present study was to demonstrate the ability of L. paracasei CNCM I-4034, B. breve CNCM I-4035 and L. rhamnosus CNCM I-4036 to inhibit the growth of $E$. coli ETEC, E. coli EPEC, Salmonella and Shigella.

\section{Materials and methods}

\section{Lactic acid bacteria strains from exclusively breastfed infants}

Three novel strains of lactic acid bacteria were isolated from the faeces of breastfed infants, and strains that demonstrated adhesion to intestinal epithelial cells and resistance to gastrointestinal conditions were identified and denominated as L. paracasei CNCM I-4034, B. breve CNCM I-4035 and L. rhamnosus CNCM I-4036, according to a previously described protocol $^{(11)}$. Briefly, twelve healthy, exclusively breastfed infants, aged 1 month, were selected for the study at the Clinic Hospital of the University of Granada. The present study was conducted according to the guidelines laid down in the Declaration of Helsinki, and all procedures involving human subjects were approved by the Ethical Committee of the University of Granada. Written informed consent was obtained from the parents after a careful explanation of the nature of the study.

\section{Isolation of supernatants from lactic acid bacteria strains}

To obtain probiotic bacteria supernatants with $1 \times$ or $10 \times$ concentrations, the bacterial strains were grown anaerobically for 17 or $24 \mathrm{~h}$ at $37^{\circ} \mathrm{C}$ in Man, Rogosa and Sharpe (lactobacilli) or Man, Rogosa and Sharpe plus cysteine (bifidobacteria) media, respectively.
The supernatants were obtained by centrifugation at $12000 \mathrm{~g}$ for $10 \mathrm{~min}$ and were then neutralised to $\mathrm{pH} 6.5$ using $\mathrm{NaOH}$ $(1 \mathrm{~m})$. To obtain the $10 \times$ supernatant, the neutralised $1 \times$ supernatant was concentrated by freeze-drying. The supernatants were sterilised by filtration through $0.22 \mu \mathrm{m}$ pore-sized filters and stored at $-20^{\circ} \mathrm{C}$ until further use.

\section{Escherichia coli ETEC, Escherichia coli EPEC, Salmonella and Shigella strains and growth conditions}

E. coli ETEC, E. coli EPEC, Salmonella typhimurium, Salmonella typhi and Shigella sonnei strains were obtained from the Spanish Type Culture Collection (CECT). We chose the following strains:

E. coli ETEC: CECT 501 and CECT 515; E. coli EPEC: CECT 727 and CECT 729; S. typhimurium CECT 443 and CECT 4594; S. typhi: CECT 725; and S. sonnei: CECT 457, CECT $4887^{\mathrm{T}}$ and CECT 413.

The E. coli and Salmonella strains were grown in tryptone soy broth and incubated aerobically for $24 \mathrm{~h}$ at $37^{\circ} \mathrm{C}$. The Shigella strains were grown in nutrient broth and incubated aerobically for $24 \mathrm{~h}$ at $37^{\circ} \mathrm{C}$.

\section{Activity of lactic acid bacteria supernatants against pathogens}

These assays were performed in polystyrene ninety-six-well (volume, $200 \mu \mathrm{l} /$ well) plates (Maxisorp). Tryptone soy (E. coli and Salmonella) or nutrient (Shigella) broth was inoculated with a $5 \%(\mathrm{v} / \mathrm{v})$ concentrated microbial cell solution and grown overnight (E. coli and Salmonella). The supernatants, with or without neutralisation, were added to a final concentration (v/v) of 1 and $4 \%$ (E. coli, Salmonella and Shigella) or just neutralised to 2 and $4 \%$ (Shigella). The ability of each strain to inhibit the pathogenic strains was evaluated by monitoring bacterial growth at $37^{\circ} \mathrm{C}$ in tryptone soy or nutrient medium in ninety-six-well plates according to the methods of Chenoll et al ${ }^{(10)}$. Bacterial growth was analysed at $620 \mathrm{~nm}$ using a Multiskan microplate reader (Thermo Fisher Scientific). In each case, the percentage of resistance was calculated by comparing the final optical densities at $620 \mathrm{~nm}$ obtained with different concentrations of supernatants with those of the corresponding control samples.

\section{Statistical analysis}

Results are expressed as mean and standard deviation. The differences between the mean values for the different treatments with lactic acid bacteria supernatants were analysed using one-way ANOVA. The least significant difference test was used for a posteriori $t$ paired comparison of the mean values. The statistical analysis was performed using Statgraphics plus (version 5.1) software (Manugistics).

\section{Results}

\section{Pathogen inhibition assays}

The L. paracasei CNCM I-4034 supernatants were specific in their effects against the pathogenic strains. The not neutralised 
$17 \mathrm{~h}$ supernatant when used at 1 and $4 \%$ concentrations inhibited the growth of S. typhi CECT 725 by 23 and $41 \%$, respectively. However, when it was neutralised, the inhibitory capacity was lost. The not neutralised $24 \mathrm{~h}$ supernatant used at 1 and $4 \%$ concentrations inhibited the growth of $S$. typhi CECT 725 by 25 and $23 \%$, respectively; when this supernatant was neutralised, growth was inhibited by 24 and $23 \%$, respectively (Fig. 1(c)). These results suggest that compounds of different nature might be present in the supernatant.

When both not neutralised supernatants were used at $4 \%$ concentration, the $17 \mathrm{~h}$ supernatant inhibited the growth of S. typhimurium CECT 443 and the $24 \mathrm{~h}$ supernatant inhibited the growth of S. typhimurium CECT 4594 by 29 and 39\%, respectively (Fig. 1(a) and (b)). The neutralised supernatants inhibited the growth of $S$. sonnei CECT 413 by $81 \%(17 \mathrm{~h})$ and $82 \cdot 10 \%(24 \mathrm{~h})$ when utilised at $4 \%$ concentration and by $32.5 \%(17 \mathrm{~h})$ when utilised at $2 \%$ concentration (Fig. 4(a)). Similar results were observed against $S$. sonnei CECT $4887^{\mathrm{T}}$, as the supernatants used at $4 \%$ concentration showed $9 \cdot 2 \%$ ( $17 \mathrm{~h}$ ) and $20.5 \%(24 \mathrm{~h})$ inhibition, and those used at $2 \%$ concentration showed $9.9 \%(24 \mathrm{~h}$ ) inhibition (Fig. 4(b)). Only the 17 h supernatant was effective against $S$. sonnei CECT 457 , as growth was inhibited by 6.3 and $25.9 \%$ when utilised at 2 and $4 \%$ concentrations, respectively (Fig. 4(c)).

When used at 1 and $4 \%$ concentrations, the not neutralised $17 \mathrm{~h} B$. breve CNCM I-4035 supernatant was able to inhibit the growth of $S$. typhi CECT 725 by 37 and $46 \%$, respectively, and by 25 and $29 \%$, respectively, when the supernatants were neutralised. Similar results were obtained with the $24 \mathrm{~h}$ supernatants at 1 and $4 \%$ concentrations, as these showed 34 and $48 \%$ inhibition, respectively, when the supernatants were not neutralised and 34 and 15\% inhibition, respectively, when the supernatants were neutralised (Fig. 2(c)). The supernatants did not inhibit the growth of E. coli EPEC CECT 727 and E. coli EPEC CECT 729 (Fig. 2(a) and (b)). However, the neutralised supernatants did inhibit the growth of $S$. sonnei CECT 457 by $19.4 \%(24 \mathrm{~h})$ when utilised at $4 \%$ concentration and by $25.8 \%(24 \mathrm{~h})$ when utilised at $2 \%$ concentration (Fig. 4(f)). The neutralised supernatants were not able to inhibit S. sonnei CECT $4887^{\mathrm{T}}$ and S. sonnei CECT 413 (Fig. 4(e) and (d), respectively).

The L. rhamnosus CNCM I-4036 not neutralised 17 and $24 \mathrm{~h}$ supernatants inhibited the growth of $S$. typhi CECT 725 by 55 and $29 \%$, respectively, but this inhibition was completely lost

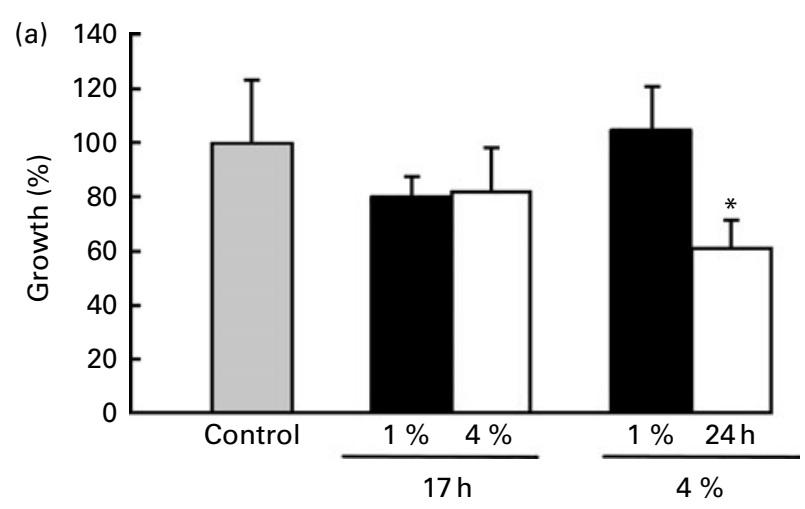

Not neutralised supernatant

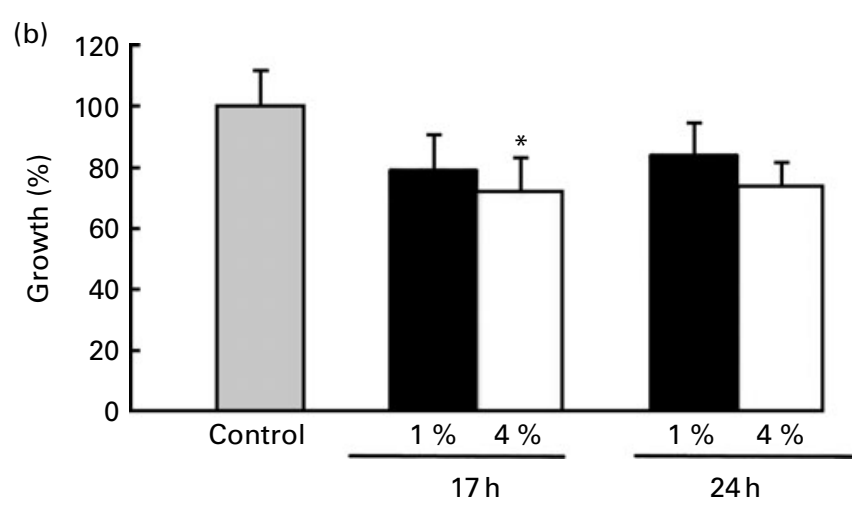

Non neutralised supernatant

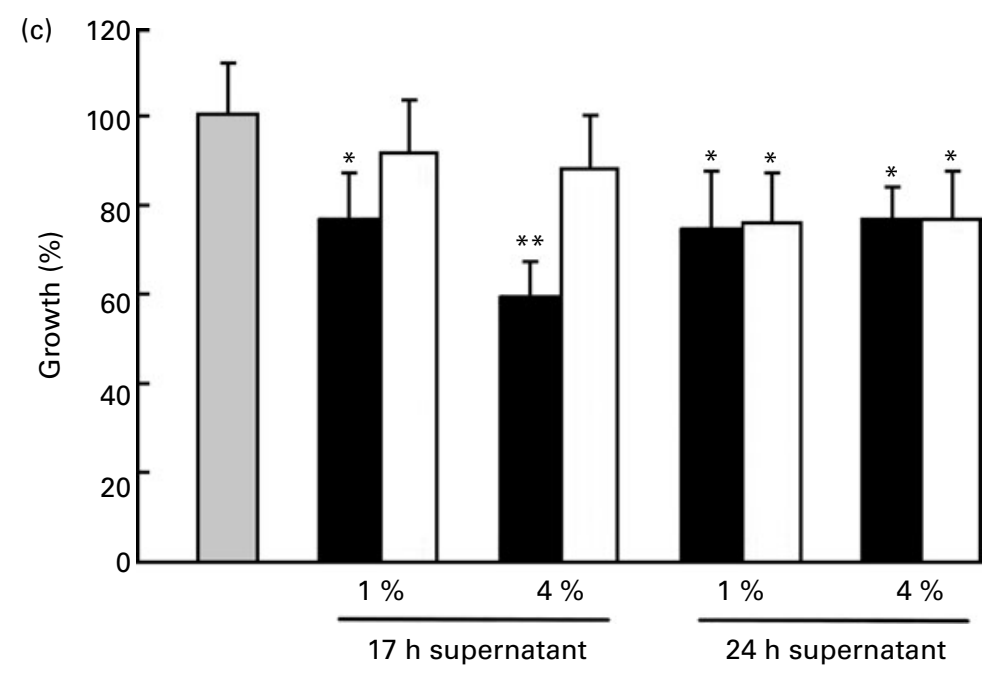

Fig. 1. Effect of 17 and $24 \mathrm{~h}$ supernatants of Lactobacillus paracasei CNCM I-4034 on growth of Salmonella typhimurium (a) CECT 4594 , (b) CECT 443 and (c) Salmonella typhi CECT 725. Values were significantly different: ${ }^{\star} P<0.05$; ${ }^{\star *} P<0.01$. $\square$, Control; $\mathbf{\square}$, not neutralised; $\square$, neutralised. 
(a)

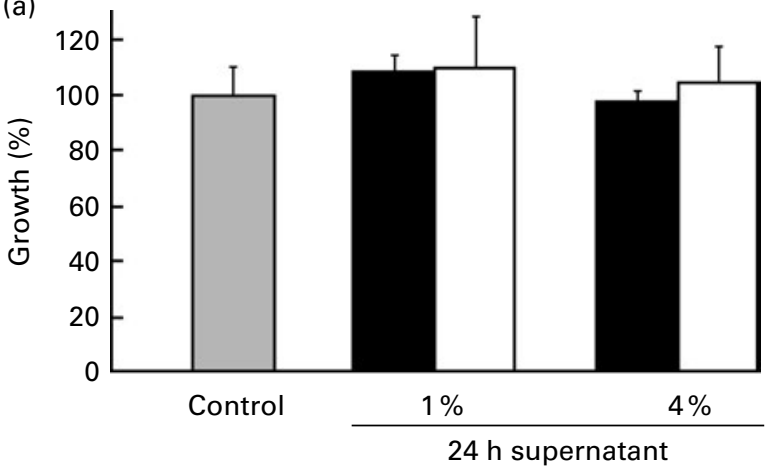

(b)

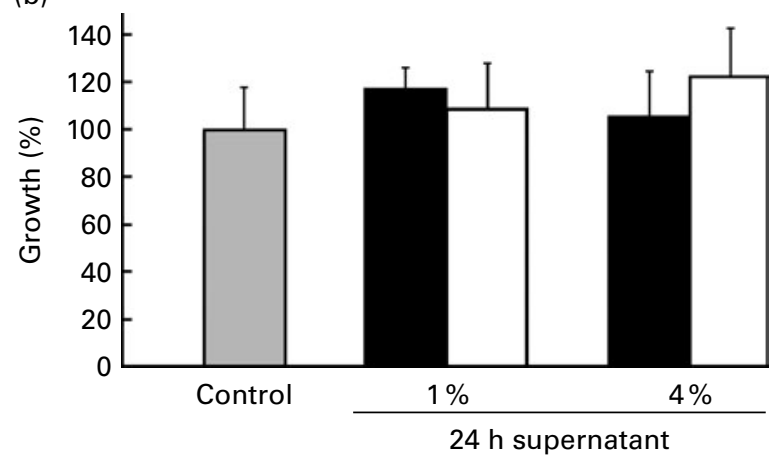

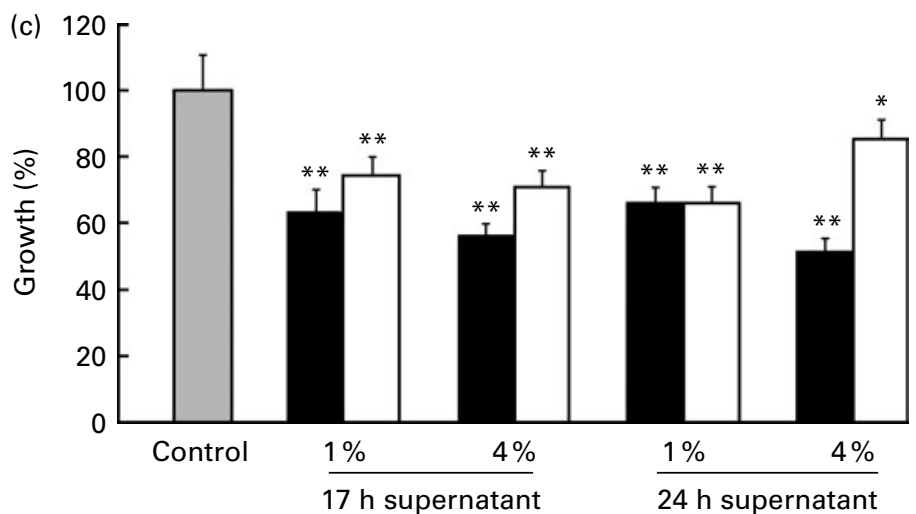

Fig. 2. Effect of supernatant of Bifidobacterium breve CNCM I-4035 on growth of Escherichia coli enteropathogenic (a) CECT 729 , (b) CECT 727 and (c) Salmonella typhi CECT 725. Values were significantly different: ${ }^{\star} P<0.05$; ${ }^{* \star} P<0.01$. $\square$, Control; $\mathbf{\square}$, not neutralised; $\square$, neutralised.

when the supernatants were neutralised (Fig. 3(a)). Similar results were observed against $E$. coli ETEC CECT 501, with 40 and $25 \%$ inhibition, and these 17 and $24 \mathrm{~h}$ supernatants also inhibited the growth of E. coli ETEC CECT 515 by 29 and $31 \%$, respectively (Fig. 3(b) and (d)). The growth of E. coli EPEC CECT 729 was only affected by the $17 \mathrm{~h}$ supernatants used at $4 \%$ concentration ( $27 \%$ inhibition, Fig. 3(c)). The neutralised supernatants did inhibit the growth of S. sonnei CECT 413 by $81 \%(17 \mathrm{~h})$ and $82 \%(24 \mathrm{~h})$ when utilised at $4 \%$ concentration, and 16.3\% (24h) inhibition was observed when utilised at $2 \%$ concentration (Fig. $4(\mathrm{~g})$ ). The supernatants were slightly effective against $S$. sonnei CECT $4887^{\mathrm{T}}$, with minor inhibitory percentages of $29 \cdot 1 \%(24 \mathrm{~h})$ when utilised at $4 \%$ concentration (Fig. 4(h)), and against S. sonnei CECT 457, showing 33.8\% (24h) inhibition when the supernatant was utilised at $4 \%$ concentration and $16 \%$ (24h) inhibition when utilised at $2 \%$ concentration (Fig. 4(i)).

\section{Discussion}

Probiotics display important characteristics that benefit human health. Although knowledge of the potential mechanisms underlying the effects of probiotics against enteropathogens is largely unknown, these mechanisms are likely multifactorial. Important mechanisms that have been shown to underlie these antagonistic effects include the reduction of luminal $\mathrm{pH}$, competition for adhesion sites and nutritional sources, secretion of antimicrobial substances, toxin inactivation and immune stimulation ${ }^{(3,14)}$. Several previous reports have indicated that lactic acids, organic acids, bacteriocins, proteases, peroxides and exopolysaccharides exert antibacterial and antifungal effects ${ }^{(3,5,15)}$. In addition, three novel probiotics (L. paracasei CNCM I-4034, B. breve CNCM I-4035 and L. rhamnosus CNCM I-4036) were previously demonstrated to resist low $\mathrm{pH}$ and high bile salt concentrations and to adhere to the colon ${ }^{(11)}$. Moreover, a safety evaluation of these probiotics, including their antibiotic resistance patterns, an assessment of certain metabolic activities (D-lactate production, bile salt deconjugation and amine biogen production) and in vivo acute ingestion profiles (immunosuppressed and immunocompetent mice), was also performed. Taken together, these previous results demonstrated that these probiotics were able to inhibit some strains of $L$. monocytogenes and the infection of human cells with rotavirus in vitro ${ }^{(11)}$

The results of the present study demonstrated that supernatants from cultures of the three novel probiotics $L$. paracase $i$ CNCM I-4034, B. breve CNCM I-4035 and L. rhamnosus CNCM I-4036 inhibited the growth of EPEC bacteria in a strainspecific manner. Tsai et al. ${ }^{(6)}$ showed a similar effect of three Lactobacillus strains against E. coli ETEC during a $20 \mathrm{~h}$ incubation, and similar results have also been obtained using organic acids ${ }^{(16,17)}$. For Bifidobacterium strains, the production of different compounds with inhibitory capacity against enteropathogen strains has also been described ${ }^{(18-20)}$. 
(a)

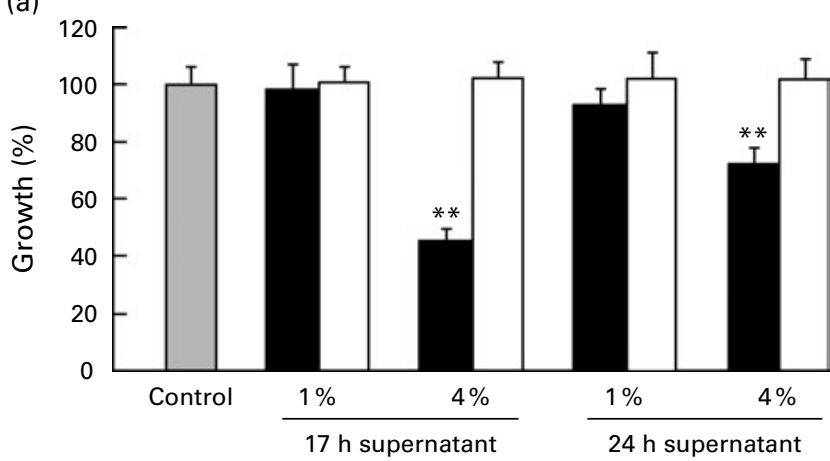

(c)

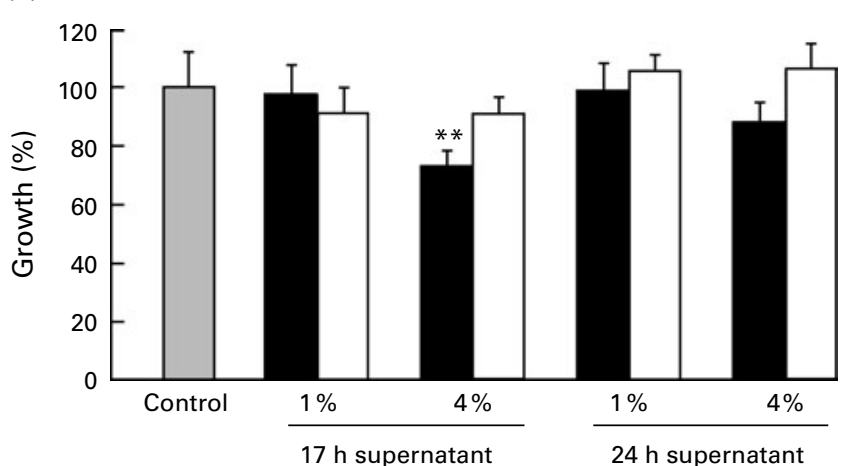

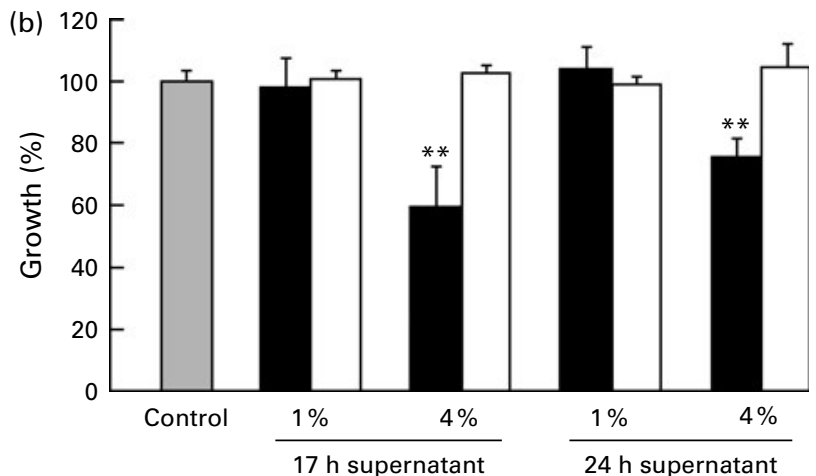

(d)

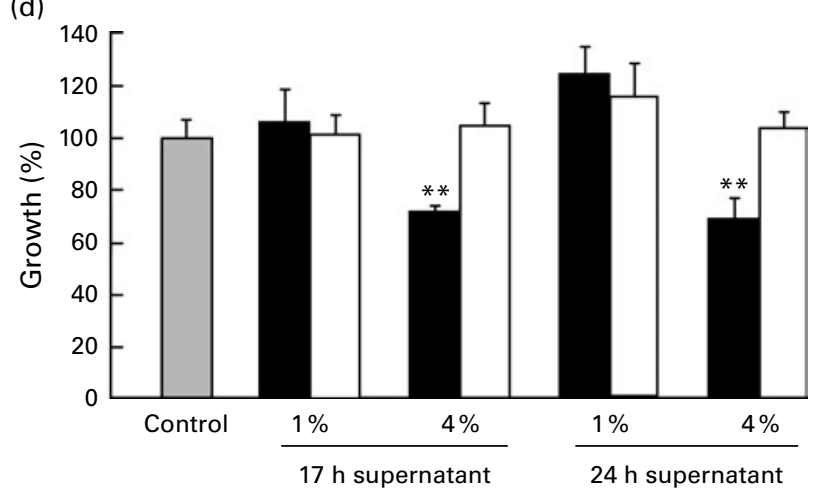

Fig. 3. Effect of supernatant of Lactobacillus rhamnosus CNCM I-4036 on growth of (a) Salmonella typhi CECT 725, (b) Escherichia coli ETEC CECT 501, (c) E. coli EPEC CECT 729 and E. coli ETEC CECT 515. ** Value was significantly different $(P<0.01)$. $\square$, Control; $\mathbf{a}$, not neutralised; $\square$, neutralised.

The L. paracasei CNCM I-4034 $17 \mathrm{~h}$ supernatants showed a differential effect against $S$. typhi CECT 725, as inhibition was observed when the supernatants were not neutralised, but these effects were lost upon neutralisation. A similar finding demonstrated that when both Lactobacillus supernatants were used against $S$. typhimurium CECT 4594, S. typhimurium CECT 443 and E. coli EPEC CECT 729, only the supernatant that was not neutralised was able to inhibit the growth of EPEC bacteria when utilised at $4 \%$ concentration. These results are consistent with those described in the study by Mauch et al. ${ }^{(15)}$, who found that the activity of the compounds produced by Lactobacillus brevis PS1 was higher at low pH values, i.e. $\mathrm{pH} \mathrm{5}$, and that this effect was partially diminished after proteolytic treatment, indicating the production of organic acid and proteinaceous compounds ${ }^{(5)}$. In another study, the total loss of antimicrobial activity at $\mathrm{pH} 7$ suggested that organic acids were involved ${ }^{(21)}$. At low $\mathrm{pH}$ values, organic acids exist in non-dissociated forms, which facilitate their penetration into the hydrophobic cell membranes of bacteria. Several reports have also shown that the major groups of inhibitory compounds produced by the probiotics include lactic acid, volatile acids and bacteriocins ${ }^{(5,15,21-23)}$.

The $B$. breve CNCM I-4035 supernatants showed a strainspecific effect on $S$. typhi CECT 725, as growth was inhibited in all cases. The nature of compounds produced by bifidobacteria differs from organic acid ${ }^{(18)}$; these substances, which are present in the supernatant, are highly effective against $S$. typhimurium and $E$. coli, and this effect has been attributed to the production of low-molecular-weight lipophilic molecules ${ }^{(19)}$.
Some authors have attributed the production of organic acid to antimicrobial mechanisms ${ }^{(24,25)}$. In addition, Fukuda et $a l .{ }^{(20)}$ found that the acetate produced by bifidobacteria improves intestinal defence against $E$. coli EHEC.

L. rhamnosus CNCM I-4036 inhibited the growth of enteropathogens by $10-50 \%$ when supernatants that were not neutralised were used; however, these effects were diminished upon neutralisation. De Keersmaecker et al. ${ }^{(26)}$ proposed that the production of lactic acid may be responsible for the antimicrobial effect of L. rhamnosus against S. typhimurium. However, we cannot disregard the possibility that this inhibitory effect was due to the added production of organic acids and bacteriocins that remain active in an acidic $\mathrm{pH}$.

The extent of the culture incubation may also serve to concentrate the inhibitory substances, which suggests that the concentration of the supernatant after fewer hours of culture may not be high enough to inhibit the growth of enteropathogens. For example, L. paracasei CNCM I-4034 $24 \mathrm{~h}$ supernatants exhibited an inhibitory effect against $S$. typhi CECT 725 , which was not affected by neutralisation; however, a different result was observed with the $17 \mathrm{~h}$ supernatants.

The antimicrobial activity of probiotics against a wide range of pathogenic micro-organisms has been observed. S. sonnei is an enteroinvasive pathogen that induces the inflammatory destruction of the intestinal epithelium, leading to acute recto-colitis and lethal complications ${ }^{(27)}$. Some authors have demonstrated the inhibition of S. sonnei using Lactobacillus supernatants that were not neutralised ${ }^{(21,28,29)}$. Zhang et al. ${ }^{(28)}$ described the inhibition of $S$. sonnei using five different 
(a)

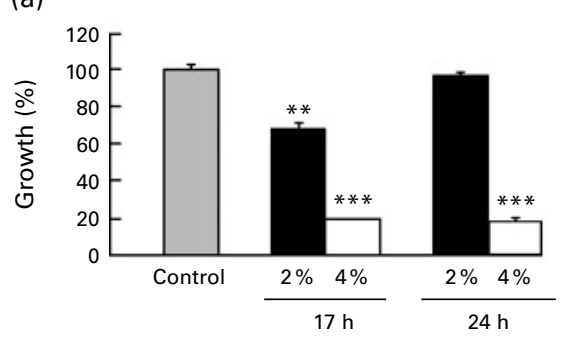

(d)

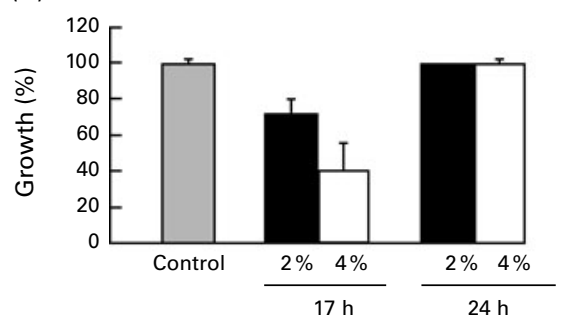

Lactobacillus paracasei CNCM I-4034

(b)

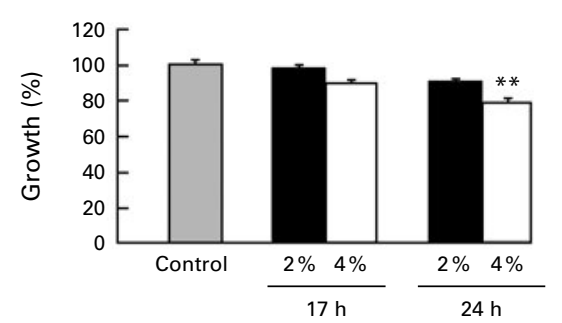

Bifidobacterium breve CNCM I-4035

(e)

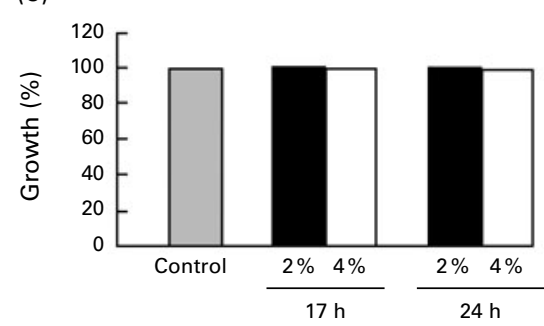

(c)

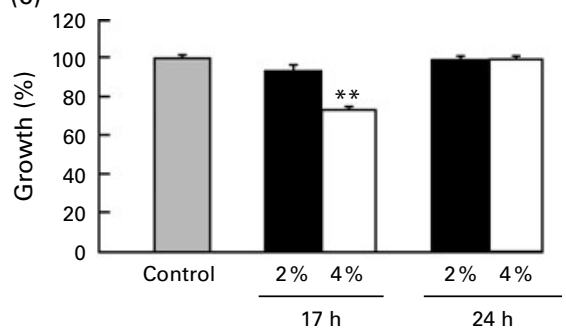

(f)

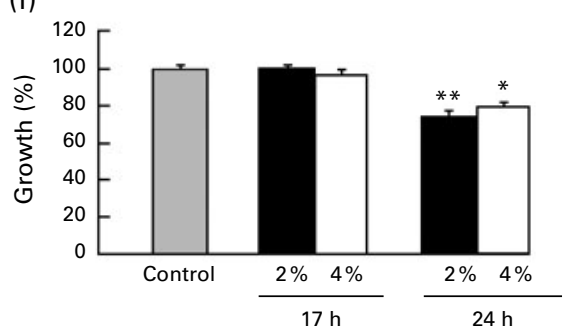

Lactobacillus rhamnosus CNCM I-4036

(g)

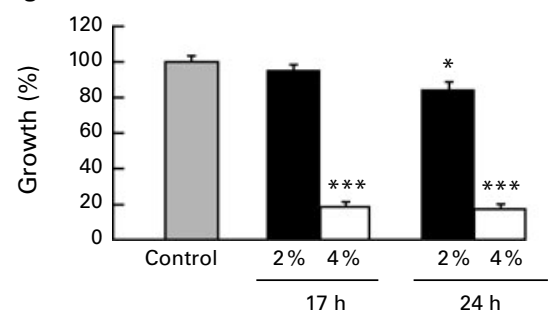

(h)

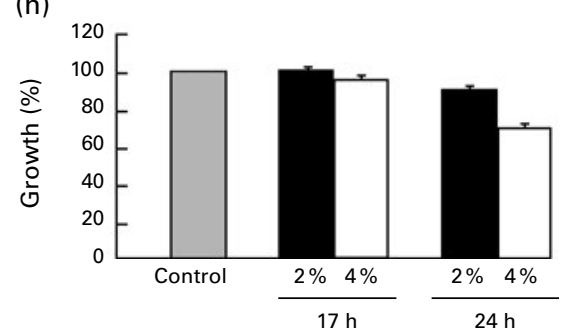

(i)

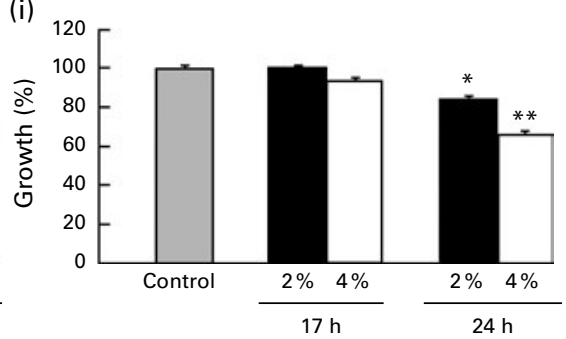

Fig. 4. Effect of 17 and $24 \mathrm{~h}$ neutralised supernatants of Lactobacillus paracasei CNCM I-4034, Bifidobacterium breve CNCM I-4035 and Lactobacillus rhamnosus CNCM I-4036 on growth of Shigella sonnei $(\mathrm{a}, \mathrm{d}, \mathrm{g})$ CECT 413 , (b, e, h) CECT $4887^{\top}$ and $(\mathrm{c}, \mathrm{f}, \mathrm{i})$ CECT 457 . Values were significantly different: ${ }^{*} P<0.05$, ${ }^{\star \star} P<0.01 ;{ }^{\star \star \star} P<0.001$. $\square$, Control; $\mathbf{\square}, 2 \% ; \square, 4 \%$.

supernatants from Lactobacillus strains, although this capacity was completely lost when the supernatants were neutralised. These results suggest that the action of organic acids is important and that bacteriocins are not involved in the antimicrobial activity of probiotics. However, different results were obtained with the strains tested in the present study, as the highest percentages of growth inhibition with $L$. paracasei CNCM I-4034 and L. rhamnosus CNCM I-4036 against $S$. sonnei CECT 413 (>80\%) were obtained with neutralised supernatants, which indicates that substances other than organic acids were involved. B. breve CNCM I-4035 did not inhibit $S$. sonnei CECT $4887^{\mathrm{T}}$ and $S$. sonnei CECT 413. Low percentages of inhibition $(<25 \%)$ were observed against S. sonnei CECT 457 , and other studies have demonstrated similar results for bifidobacteria strains $^{(30)}$.

A mixture of organic acids and proteinaceous compounds may affect the growth of EPEC bacteria in a strain-specific manner. Furthermore, due to the reduction in $\mathrm{pH}$, this antimicrobial activity may be attributed to the presence of non-dissociated forms of acids. However, neutralised supernatants were shown to both inhibit and stimulate the growth of enteropathogens in a strain-specific manner, although the role of additional substances is possible. Taken together, the present results demonstrated that the inhibitory effects of probiotic supernatants were highly strain specific, and similar results have been previously described by other authors ${ }^{(31,32)}$.

In conclusion, in the present study, we showed that supernatants harvested at different culture times from three novel probiotic strains can inhibit the growth of selected strains of E. coli, Salmonella and Shigella. These results suggest that these probiotic strains may produce substances that are either permissive or harmful to enteropathogens, depending on the neutralisation, concentration and culture time of the supernatants. These compounds could be organic acid and/ or bacteriocins and are strain specific. Reactome analysis are ongoing to determine the nature of these substances.

\section{Acknowledgements}

S. M.-Q. and E. C. carried out the majority of the experiments and were responsible to write the first draft of the manuscript. S. M.-Q. and M. B.-B. were involved in the experiments with E. coli and Salmonella and E. C. with those of S. sonnei. C. G.-L. and S. G. helped in the studies of bacteria competition. J. P.-D. was involved in the evaluation and preparation of the manuscript. E. M., M. J. B. and F. R. participated in the 
process of strain characterisation. D. R. and A. G. were the scientist leaders of the present work and contributed to the design and supervision of the experiments and results; they also were involved in writing the manuscript. This work was supported by the HERO Group trough its company HERO Spain S.A. (contract no. 3143 signed with the Fundación General Universidad de Granada Empresa contract and a private contract signed with the Spanish Biotechnology Company Biópolis S.L.). Hero Spain S.A. in turn was funded by the CDTI, Spanish Ministry of Health. C. G.-L. is a recipient of a postdoctoral fellowship from Plan Propio of the University of Granada. E. M., M. J. B. and F. R. are members of the Department of Research \& Development, Hero Institute for Infant Nutrition. This Institute forms part of the food company HERO with headquarters in Switzerland. E. C., S. G. and D. R. are members of Biópolis S.L. (Spain), a spin-off of the High Scientific Research Council (Consejo Superior de Investigaciones Cientificas), Ministry of Education, Spain. No other authors declare conflict of interest.

\section{References}

1. FAO/WHO (2002) Guidelines for the Evaluation of Probiotics in Food. Working Group Report. London, Ontario: Food and Health Agricultural Organisation of the United Nations - World Health Organisation.

2. Minocha A (2009) Probiotics for preventive health. Nutr Clin Pract 24, 227-241.

3. Servin AL (2004) Antagonistic activities of lactobacilli and bifidobacteria against microbial pathogens. FEMS Microbiol Rev 28, 405-440.

4. Collado MC, Gueimonde M, Salminem S (2010) In: Bioactive foods in promoting bealth, probiotics and prebiotics, 1st ed., pp. 353-370 [Watson RR and Preedy VR, editors]. London, UK: Academic Press, Elsevier.

5. Wang H, Yan Y, Wang J, et al. (2012) Production and characterization of antifungal compounds produced by Lactobacillus plantarum IMAU10014. PLoS One 7, e29452.

6. Tsai CC, Lin PP \& Hsieh YM (2008) Three Lactobacillus strains from healthy infant stool inhibit enterotoxigenic Escherichia coli grown in vitro. Anaerobe 14, 61-67.

7. Tsai CC, Hsih HY, Chiu HH, et al. (2005) Antagonistic activity against Salmonella infection in vitro and in vivo for two Lactobacillus strains from swine and poultry. Int J Food Microbiol 102, 185-189.

8. de LeBlancade M, Castillo NA \& Perdigon G (2010) Antiinfective mechanisms induced by a probiotic Lactobacillus strain against Salmonella enterica serovar Typhimurium infection. Int J Food Microbiol 138, 223-231.

9. Dobson A, Cotter PD, Ross RP, et al. (2012) Bacteriocin production: a probiotic trait? Appl Environ Microbiol 78, 1-6.

10. Chenoll E, Casinos B, Bataller E, et al. (2011) Novel probiotic Bifidobacterium bifidum CECT 7366 strain active against the pathogenic bacterium Helicobacter pylori. Appl Environ Microbiol 77, 1335-1343.

11. Muñoz-Quezada S, Chenoll E, Vieites JM, et al. (2012) Isolation, identification and characterization of three novel probiotic strains (Lactobacillus paracasei CNCM I-4034, Bifidobacterium breve CNCM I-4035 and Lactobacillus rhamnosus CNCM I-4036) from the faeces of exclusively breastfed infants. Br J Nutr. (doi:10.1017/S0007114512005211).

12. Gribble KD (2011) Mechanisms behind breastmilk's protection against, and artificial baby milk's facilitation of, diarrhoeal illness. Breastfeed Rev 19, 19-26.
13. Le Huërou-Luron I, Blat S \& Boudry G (2010) Breast- $v$. formula-feeding: impacts on the digestive tract and immediate and long-term health effects. Nutr Res Rev 23, 23-36.

14. Fooks LJ, Fuller R \& Gibson GR (1999) Prebiotics, probiotics and human gut microecology. Int Dairy J 9, 53-61.

15. Mauch A, Dal Bello F, Coffey A, et al. (2010) The use of Lactobacillus brevis PS1 to in vitro inhibit the outgrowth of Fusarium culmorum and other common Fusarium species found on barley. Int J Food Microbiol 141, 116-121.

16. Bernet-Camard MF, Liévin V, Brassart D, et al. (1997) The human Lactobacillus acidophilus strain LA1 secretes a nonbacteriocin antibacterial substance(s) active in vitro and in vivo. Appl Environ Microbiol 63, 2747-2753.

17. Coconnier MH, Liévin V, Bernet-Camard MF, et al. (1997) Antibacterial effect of the adhering human Lactobacillus acidophilus strain LB. Antimicrob Agents Chemother 41, 1046-1052.

18. Collado MC, Hernández M \& Sanz Y (2005) Production of bacteriocin-like inhibitory compounds by human fecal Bifidobacterium strains. J Food Prot 68, 1034-1040.

19. Liévin V, Peiffer I, Hudault S, et al. (2000) Bifidobacterium strains from resident infant human gastrointestinal microflora exert antimicrobial activity. Gut 47, 646-652.

20. Fukuda S, Toh H, Hase K, et al. (2011) Bifidobacteria can protect from enteropathogenic infection through production of acetate. Nature 469, 543-547.

21. Lin WH, Yu B, Jang SH, et al. (2007) Different probiotic properties for Lactobacillus fermentum strains isolated from swine and poultry. Anaerobe 13, 107-113.

22. Millette M, Luquet FM \& Lacroix M (2007) In vitro growth control of selected pathogens by Lactobacillus acidophilusand Lactobacillus casei-fermented milk. Lett Appl Microbiol 44, 314-319.

23. Lee H, Yoon H, Ji Y, et al. (2011) Functional properties of Lactobacillus strains isolated from kimchi. Int $J$ Food Microbiol 145, 155-161.

24. Makras L \& de Vuyst L (2006) The in vitro inhibition of Gram-negative pathogenic bacteria by bifidobacteria is caused by the production of organic acids. Int Dairy $J \mathbf{1 6}$, 1049-1057.

25. Morita H, Hashimoto H, Hosoda M, et al. (2002) Intestinal Bifidobacterium species induce varying cytokine production. J Allergy Clin Immunol 109, 1035-1036.

26. De Keersmaecker SC, Verhoeven TL, Desair J, et al. (2006) Strong antimicrobial activity of Lactobacillus rhamnosus GG against Salmonella typhimurium is due to accumulation of lactic acid. FEMS Microbiol Lett 259, 89-96.

27. Niyogi SK (2005) Shigellosis. J Microbiol 43, 133-143.

28. Zhang Y, Zhang L, Du M, et al. (2011) Antimicrobial activity against Shigella sonnei and probiotic properties of wild lactobacilli from fermented food. Microbiol Res 167, 27-31.

29. Jara S, Sánchez M, Vera R, et al. (2011) The inhibitory activity of Lactobacillus spp. isolated from breast milk on gastrointestinal pathogenic bacteria of nosocomial origin. Anaerobe 17, 474-477.

30. Hütt P, Shchepetova J, Lõivukene K, et al. (2006) Antagonistic activity of probiotic lactobacilli and bifidobacteria against entero- and uropathogens. J Appl Microbiol 100, $1324-1332$.

31. Gueimonde M, Jalonen L, He F, et al. (2006) Adhesion and competitive inhibition and displacement of human enteropathogens by selected lactobacilli. Food Res Int 39, 467-471.

32. Salminen S, Nybom S, Meriluoto J, et al. (2010) Interaction of probiotics and pathogens - benefits to human health? Curr Opin Biotechnol 21, 157-167. 